\title{
Effects of exercise training on heart rate variability in children and adolescents with pulmonary arterial hypertension: a pilot study
}

\author{
Jannos Siaplaouras ${ }^{1}$, Marc Frerix $^{2}$, Anita Apitz ${ }^{1}$, David Zöller ${ }^{3}$, Christian Apitz ${ }^{1,3}$ \\ ${ }^{1}$ Division of Pediatric Cardiology, University Childrens Hospital Ulm, Ulm, Germany; ${ }^{2}$ Department of Internal Medicine and Rheumatology, Justus- \\ Liebig-University Giessen, Giessen, Germany; ${ }^{3}$ Pediatric Heart Center, Justus-Liebig-University Giessen, Giessen, Germany \\ Contributions: (I) Conception and design: J Siaplaouras, A Apitz, D Zöller, C Apitz; (II) Administrative support: A Apitz, D Zöller, C Apitz; (III) \\ Provision of study materials or patients: C Apitz; (IV) Collection and assembly of data: D Zöller, A Apitz, J Siaplaouras; (V) Data analysis and \\ interpretation: M Frerix, J Siaplaouras; (VI) Manuscript writing: All authors; (VII) Final approval of manuscript: All authors. \\ Correspondence to: Prof. Dr. Christian Apitz. Division of Pediatric Cardiology, University Childrens Hospital Ulm, Eythstr. 24; D-89075 Ulm, \\ Germany. Email: Capitz@aol.com.
}

Background: Pulmonary arterial hypertension (PAH) is often associated with cardiac autonomic dysfunction, and heart rate variability (HRV) as marker of cardiac autonomic function is even related to disease severity. Knowledge about the effects of physical activity on HRV is limited in these patients. We aimed to assess whether HRV parameters can be influenced by a supervised exercise training program and whether respective changes are related to levels of activity.

Methods: Six children and adolescents with moderate PAH (3 female, mean age 15.0 \pm 4.4 years; mean pulmonary to systemic arterial pressure ratio $0.5 \pm 0.2)$ performed supervised endurance and resistance training for 16 weeks. PAH-specific targeted medication remained unchanged during the study period. HRV was assessed before training and after 16 weeks of training by the use of ECG Move accelerometers. HRV indices included: standard deviation of normal-to-normal (NN) intervals (SDNN), square root of the mean of the sum of the squares of differences between successive NN-intervals (RMSSD), proportion of the number of pairs of successive normal-to-normal intervals that differ by more than 50 ms divided by total number of normal-to-normal interval (pNN50), and the Baevsky stress index (BSI) calculated by the histogram method.

Results: Before and after the training program, SDNN, RMSSD and pNN50 correlated with the level of physical activity. Mean values of SDNN, RMSSD and pNN50 did not change significantly due to the training program. Notably, activity level depending SDNN increased markedly after the exercise program (during activity $+12.4 \%$, at rest $+6.6 \%$ and reclining $+8.1 \%$, Hedge's $g$ of $0.28,0.14$ and 0.27 , respectively). BSI decreased during activity due to the training program reflecting a reduction of stress level (Hedge's $g$ -0.87 indicating an effect of clinical relevance).

Conclusions: According to the experience of this pilot study a workout program of 16 weeks revealed an activity level dependent effect on parameters of autonomic cardiac function in children and adolescents with PAH. This mechanism might contribute to the positive effects of exercise training in patients with PAH.

Keywords: Heart rate variability (HRV); stress related cardiac autonomic regulation; exercise training; congenital heart disease; pulmonary hypertension

Submitted Feb 21, 2020. Accepted for publication Jul 09, 2020.

doi: $10.21037 / \mathrm{cdt}-20-263$

View this article at: http://dx.doi.org/10.21037/cdt-20-263 


\section{Introduction}

Pulmonary arterial hypertension (PAH) is a rare and complex disease characterised by vasoconstriction and progressive remodelling of the pulmonary arterial wall leading to increased pulmonary vascular resistance (PVR) and right heart failure. Despite recent advances in PAHtargeted therapies and evolving treatment guidelines, the prognosis of PAH is still very unfavorable in children (1-6).

Little is known about the diagnostic and prognostic implication of cardiac autonomic function and the effects of exercise in children with $\mathrm{PAH}$ and their underlying mechanisms (7-15).

Impaired cardiac autonomic regulation is not uncommon in PAH patients (16) and seems to be related to disease severity and prognosis $(17,18)$. In PAH patients, mean heart rate is significantly higher and heart rate variability (HRV) is decreased resulting in a higher burden of arrhythmias (19). Dysregulation is associated with increased mortality risk in healthy adults and in patients with PAH (20).

Recently, exercise training as add-on to medical treatment has shown to have beneficial effects on symptoms and hemodynamic function in pulmonary hypertension (7).

Regular exercise training is thought to modify cardiac autonomic control although there is a heritable component of heart rate regulation and response to exercise (21). Individuals who exercise regularly generally have higher HRV than individuals with sedentary lifestyle (22). Timedomain measurements correlate with aerobic fitness (23). Changes in daily step count and time spent physically active correlate with 6 minutes walk distance suggesting that accelerometry may be a useful monitoring tool (24-26).

Interestingly, we were previously able to show that an exercise training in PAH patients as add-on to optimized medical therapy resulted in improvement of chronotropic competence and a significant increase of chronotropic index (27).

According to these results we hypothesized that cardiac autonomic regulation might be positively influenced by exercise training and that changes might also be activity level dependent. We therefore performed a pilot study in children and adolescents with PAH using accelerometry before and at the end of a 16-weeks exercise program to assess activity level depending training-related effects on HRV.

\section{Methods}

\section{Study population and design}

Children and adolescents with non-severe PAH were offered to participate in this prospective study. Diagnosis was previously established by cardiac catheterization including testing for pulmonary vasoreactivity prior to the study. All patients were stable under optimized advanced PAH specific therapy for a minimum of 3 months before entering the study. Medication remained unchanged during the complete study period.

The study was conducted in accordance with the Declaration of Helsinki (as revised in 2013). This study was reviewed and approved by the Ethical Review board of the Justus-Liebig-University Giessen (approval number 219/13). All patients enrolled completed the informed consent form.

\section{Cardiopulmonary exercise testing}

To individualize the workout and for safety reasons cardiopulmonary exercise testing was performed on each patient before entering the study and after completion of the training. All patients underwent treadmill testing according to a modified Bruce protocol as described previously (28).

\section{Exercise training}

For a total of 16 weeks patients performed a supervised endurance and strength workout programm in an ambulatory setting.

The endurance training consisted of a heart rate controlled bicycle ergometer training, performed twice a week, starting with a low workload (20-25 W) for 20 to $25 \mathrm{~min} / \mathrm{d}$, achieving approximately $50 \%$ to $69 \%$ of the heart rate they had reached during peak oxygen uptake at the initial exercise test. The training intensity was increased (e.g., up to a maximum of $70 \mathrm{~W}$ ) with respect to the individual tolerability and improvement, and limited by peak heart rate (not more than $140 \mathrm{bpm}$ ), and subjective physical exertion. Training duration was limited to a maximum of 80 minutes weekly. Furthermore, a set of seven different resistance band workouts have been performed on two separate days apart from the bicycle training twice a week. All patients were advised to avoid heavy exercise. Resistance band workouts have been practised with support of local physiotherapists and further assisted by web-based training films and limited to $10-15$ repetitions and 2-3 sets. During the home-based exercise training, all patients were asked to keep in close contact with the physicians of the training program, and to complete a short online-questionnaire at 
each day of training. Furthermore, the amount of training at home and patient well-being was supervised by phone calls every 2 weeks by members of the study team.

\section{HRV and activity measurements}

Analysis of HRV has become a standard in the estimation of cardiac autonomic regulation, with multiple indices derived from HRV being routinely used. HRV provides an understanding of sympathetic and parasympathetic nervous system interaction, the so-called sympathovagal balance. HRV can be assessed either as a time domain analysis or frequency domain analysis. Basis of every calculation is the difference in time between two consecutive QRS-complexes in the obtained electrocardiogram (ECG) at rest.

Time domain measures are simply to calculate, frequency domain analyses are due to non-overlapping frequency bands well-equipped to discriminate between the contributions of the sympathetic and parasympathetic nervous system.

In this study, heart rate and parameters of HRV were therefore assessed by accelerometry using ECG Move Accelerometer (Movisens GmbH, Karlsruhe, Germany). The movisens activity sensor consists of a three-axial acceleration sensor (weight $32 \mathrm{~g}$, size $5.0 \times 3.6 \times 1.7 \mathrm{~cm}^{3}$ ) which was attached with adhesive electrodes on the chest underneath the clothes with direct skin contact. The sensor collected single channel ECG data with a resolution of 12 bits and a sampling rate of $1,024 \mathrm{~Hz}$.

Accelerometric measurements were performed on three consecutive days during the working week at baseline before start of training and after 16 weeks of training in each patient. The movisens DataAnalyzer software was used to convert the ECG signals into HRV indices.

The time domain HRV indices included: (I) standard deviation of normal-to-normal (NN) intervals (SDNN); (II) square root of the mean of the sum of the squares of differences between successive NN-intervals (RMSSD); (III) proportion of the number of pairs of successive normalto-normal intervals that differ by more than $50 \mathrm{~ms}$ divided by total number of normal-to-normal interval (pNN50); and (IV) the Baevsky stress index (BSI) calculated by the histogram method.

Calculation of frequency domain HRV measures employed power spectral density characteristics of cardiointervals using Fourier transformation and calculated the following parameters from the spectrum: HRV low frequency (LF) power (describes the spectral power in the frequency band between 0.04 and $0.15 \mathrm{~Hz}$ ), HRV high frequency (HF) power (describes the spectral power in the frequency band between 0.15 and $0.4 \mathrm{~Hz}$ ), and the LF/HF ratio as an index of vasosympathetic interaction.

\section{Statistical analysis}

Values of continuous variables were presented as mean \pm standard deviation. Values before and after exercise were compared by the paired samples $t$-test. Differences have been visualized by Dependent sample difference assessment plots. $95 \%$ confidence intervals using the $\mathrm{t}$-distribution and Cohen's d effect size applying Hedge's correction for a paired $t$-test in small samples (= Hedge's $\mathrm{g}$ ) were reported to explore the magnitude of potential clinical relevant changes. A change of at least medium effect size (Hedge's $g$ $>0.5$ ) was considered to indicate potential clinical relevance. Analysis was performed using $\mathrm{R}$ software version 3.2.0 (R Foundation for Statistical Computing, Vienna, Austria), $\mathrm{R}$ package granovaGG version 1.4 .0 and $\mathrm{R}$ package effsize version 0.8.0 (29,30).

\section{Results}

\section{Study population}

Six children and adolescents with PAH (3 female, mean age $15.0 \pm 4.4$ years) were included in this pilot study. Baseline characteristics of the patients including demographic data, diagnosis, PAH specific drug therapy, and hemodynamic data are displayed in Table 1 . None of the patients received treatment with Betablockers or other heart-rate influencing drugs. According to the WHO classification five patients (83.3\%) were in functional class II and only one (16.7\%) was in functional class III. Catheterization data showed a mean pulmonary artery pressure (mPAP) of $42.8 \pm 15.7 \mathrm{mmHg}$, and an indexed PVR of $9.4 \pm 5.4 \mathrm{WU} \cdot \mathrm{m}^{2}$ (Table 1).

\section{Effects on cardiopulmonary exercise testing}

All patients tolerated the workout program well without any adverse events. After 16 weeks of supervised training patients showed improved exercise capacity. Both, loading time (baseline 10:24 $\pm 2: 01$; after training 11:46 $\pm 2: 27$ minutes) and treadmill distance (baseline 563.54 \pm 169.79 ; after training $692.62 \pm 232.14$ meters) increased clinically relevant (Hedge's $g=0.59$ ).

No relevant change in peak oxygen consumption (peak 
Table 1 Baseline characteristics (values are mean \pm standard deviation)

\begin{tabular}{|c|c|}
\hline Characteristics & Outcome \\
\hline \multicolumn{2}{|l|}{ Study population } \\
\hline Male/female & $3 / 3$ \\
\hline Age (years) & $15.0 \pm 4.4$ \\
\hline Height (cm) & $162.4 \pm 16.9$ \\
\hline Weight (kg) & $64.6 \pm 33.4$ \\
\hline \multicolumn{2}{|l|}{ WHO functional class, No. (\%) } \\
\hline II & $5(83.3 \%)$ \\
\hline III & $1(16.7 \%)$ \\
\hline \multicolumn{2}{|l|}{ Diagnosis } \\
\hline Idiopathic pulmonary arterial hypertension & $5(83.3 \%)$ \\
\hline PAH-CHD (recurrent after shunt closure) & $1(16.7 \%)$ \\
\hline \multicolumn{2}{|l|}{ Catheterization data } \\
\hline Right atrial pressure $(\mathrm{mmHg})$ & $6.0 \pm 2.2$ \\
\hline Mean pulmonary arterial pressure $(\mathrm{mmHg})$ & $42.8 \pm 15.7$ \\
\hline Diastolic pulmonary arterial pressure $(\mathrm{mmHg})$ & $28.2 \pm 12.6$ \\
\hline Mean systemic arterial pressure $(\mathrm{mmHg})$ & $83.3 \pm 11.2$ \\
\hline mPAP/SAP & $0.5 \pm 0.2$ \\
\hline Pulmonary arterial wedge pressure $(\mathrm{mmHg})$ & $9.5 \pm 1.9$ \\
\hline Pulmonary vascular resistance index $\left(\mathrm{WU} \cdot \mathrm{m}^{2}\right)$ & $9.4 \pm 5.4$ \\
\hline \multicolumn{2}{|l|}{$\mathrm{PAH}$-targeted medication } \\
\hline Endothelin receptor antagonists & $4(66.7 \%)$ \\
\hline Phosphodiesterase-5-inhibitors & $3(50 \%)$ \\
\hline Calcium channel blockers & $3(50 \%)$ \\
\hline \multicolumn{2}{|l|}{ Combination therapy } \\
\hline Monotherapy & 2 (33.3\%) \\
\hline Dual therapy & $4(66.7 \%)$ \\
\hline
\end{tabular}

$\mathrm{PAH}$, pulmonary arterial hypertension; mPAP, mean pulmonary arterial pressure; CHD, congenital heart disease; mSAP, mean systemic arterial pressure; WU, Wood units.

VO2 at baseline $1,717.00 \pm 639 \mathrm{~mL} / \mathrm{kg} / \mathrm{min}$, after training $1,677.67 \pm 598 \mathrm{~mL} / \mathrm{kg} / \mathrm{min}$ ) could be observed, while oxygen consumption at the anaerobic threshold improved clinically relevant from $1,328.83 \pm 483$ to $1,403.67 \pm 486 \mathrm{~mL} / \mathrm{kg} / \mathrm{min}$ (Hedge's $g=0.5$ ). Maximum heart rate increased from $175.2 \pm 15.7$ to $181.7 \pm 13.1$ beats per minute (Hedge's $g=0.82$ ) (Table 2), while mean heart rate remained unchanged (75.98 vs. $75.77 \mathrm{bpm})$. Accelerometric assessed heart rate at rest and during activities of daily living did not reveal significant changes due to the workout program (percentage of change $-0.6 \%$ resp. $-3.4 \%$ ) (Table 3).

\section{Effects on SDNN, RMSSD and pNN50}

At baseline and after the training program, SDNN, RMSSD and pNN50 were related to the extent of activity. Mean values of SDNN, RMSSD and pNN50 did not show significant changes due to the training program. Notably, SDNN showed an activity level depending increase due to the exercise program (during activity $+12.4 \%$, at rest $+6.6 \%$ and reclining $+8.1 \%$, Hedge's $g$ of $0.28,0.14$ and 0.27 , respectively) (Figure 1). There were no relevant activity level depending changes of RMSSD and pNN50 values.

\section{Effects on BSI}

BSI was positively related to the extent of physical activity at baseline and after the training program (baseline: activity $305.76 \pm 132.07$, rest $179.57 \pm 87.55$ and reclining $115.29 \pm 14.00)$. BSI values decreased due to the training program reflecting a reduction of stress level (after training: activity $-34.3 \%$, rest $-15.9 \%$, reclining $-10.1 \%$ ). The benefit of the training program was particular clinically meaningful during activities of daily living (Hedge's $g$-0.87) (Figure 2).

\section{Effects on frequency domain HRV measures}

HRV LF and HF power bands were related to the extent of physical activity (Table 3). LF/HF ratio was also related to activity and increased from $1.0 \pm 0.53$ while reclining to $2.53 \pm 0.82$ during physical activity reflecting activity-related increased LF power. Remarkably, LF/HF ratio further increased due to the training program $(+24.3 \%$, Hedge's $g=0.64)$.

\section{Discussion}

To our knowledge, this pilot study is the first, which assessed cardiac autonomic regulation by accelerometry allowing an estimation of activity level depending interactions between the parasympathetic and sympathetic regulatory influences on the cardiovascular system after 16 weeks of supervised exercise training.

Patients with $\mathrm{PAH}$ frequently suffer from exercise limitation, while the reasons for this appear to be 
Table 2 Training effects on cardiopulmonary exercise testing (values are mean \pm standard deviation)

\begin{tabular}{lcccccc}
\hline Variable & Baseline & After training & $\begin{array}{c}\text { Mean } \\
\text { difference }\end{array}$ & $95 \% \mathrm{Cl}$ & $\begin{array}{c}\text { Change } \\
\text { in } \%\end{array}$ & Effect size, Hedge's g (95\% Cl) \\
\hline Loading time $(\mathrm{min})$ & $10: 24 \pm 2: 01$ & $11: 46 \pm 2: 27$ & $1: 22 \pm 1: 56$ & $-0: 40$ to $3: 24$ & $13.1 \%$ & $0.59(-0.24$ to 1.42$)$ \\
Treadmill distance $(\mathrm{m})$ & $563.54 \pm 169.79$ & $692.62 \pm 232.14$ & $129.97 \pm 184.43-64.47$ to 322.62 & $22.9 \%$ & $0.59(-0.24$ to 1.42$)$ \\
Heart rate at rest $(\mathrm{L} / \mathrm{min})$ & $92.50 \pm 17.93$ & $91.00 \pm 12.12$ & $-1.50 \pm 8.76$ & -10.69 to 7.69 & $-1.6 \%$ & $-0.14(-0.91$ to 0.63$)$ \\
Heart rate $\mathrm{max}(\mathrm{L} / \mathrm{min})$ & $175.17 \pm 15.68$ & $181.67 \pm 13.06$ & $6.50 \pm 6.69$ & 0.52 to 13.52 & $3.7 \%$ & $0.82(-0.07$ to 1.70$)$ \\
VO2@AT $(\mathrm{mL} / \mathrm{kg} / \mathrm{min})$ & $1,328.83 \pm 483.79$ & $1,403.67 \pm 486,64$ & $74.83 \pm 127.21$ & -58.66 to 208.33 & $5.6 \%$ & $0.50(-0.32$ to 1.31$)$ \\
VO2max $(\mathrm{mL} / \mathrm{kg} / \mathrm{min})$ & $1,717.00 \pm 639.01$ & $1,677.67 \pm 598.01$ & $39.33 \pm 101.16$ & -146.54 to 67.88 & $-2.3 \%$ & $-0.32(-1.11$ to 0.46$)$ \\
Oxygen pulse $(\mathrm{mL})$ & $9.73 \pm 3.09$ & $9.22 \pm 3.10$ & $-0.52 \pm 0.40$ & -0.93 to -0.10 & $-5.5 \%$ & $-1.1(-2.06$ to -0.13$)$ \\
EqO2 $\mathrm{max}$ & $35.85 \pm 5.89$ & $40.75 \pm 5.47$ & $4.90 \pm 3.55$ & 1.18 to 8.62 & $13.7 \%$ & $1.16(0.17$ to 2.16$)$ \\
EqCO2 $\mathrm{max}$ & $34.42 \pm 4.43$ & $35.58 \pm 4.19$ & $1.17 \pm 4.58$ & -3.64 to 5.98 & $3.4 \%$ & $0.21(-0.56$ to 0.99$)$ \\
\hline
\end{tabular}

@AT, at the anaerobic threshold.

multifactorial beyond right ventricular dysfunction. Exercise training improves the function of different body organs such as heart, lung and skeletal muscle, and can modulate several mechanisms acknowledged in PAH pathophysiology such as oxidative stress, inflammation, vasoconstriction, vascular remodelling, and thrombosis. It has previously been shown that chronotropic incompetence and autonomic dysregulation may also play an important role in exercise limitation and can be improved by exercise training $(15,17,18)$.

Different mechanisms have been discussed in the literature, that contribute to impaired HRV in patients with PAH. For example, stretching of the sinoatrial node or chronic increased sympathetic nerve activity may result in a reduction of HRV (31). Another reason might be a down-regulation of beta-adrenergic receptors which has been previously shown to impact HRV in left and right ventricular failure (32).

In this study the analysis of parameters of cardiac autonomic regulation revealed clinically remarkable effects with a shift towards less sympathetic activity after 16 weeks of exercise training, which was more obvious under physical activity. The most distinct training effects have been detected for the BSI, which originates from Russian space medicine and describes the stress state of a person (33). The Baevsky index reflects the rhythm stabilisations and disorder reductions in the cardio-interval length calculated by the histogram of the heart rhythm distribution curve. The effects on the Baevsky index in our study were more obvious during physical activity assuming less sympathetic stress presumably due to the training effect.
SDNN is the "gold standard" in the evaluation of HRV and is a strong predictor of morbidity and mortality (34). Interestingly, SDNN correlated with the activity level and increased due to the training program, which can be interpreted as improvement of HRV since it has previously been shown that lower SDNN is correlated with a more severe disease state in PAH patients $(17,18)$. The effect was even more visible during intense physical activity. Also, RMSSD and pNN50 correlated before and after the training with the extent of physical activity.

The effects on frequency domain HRV measures, which is mainly determined by the parasympathetic system (35), revealed also interesting findings, most notably for the ratio of HRV LF and HF power bands. While power of the HF component can be taken as an index of cardiac parasympathetic tone and the LF component as a marker of cardiac sympathetic outflow, the LF/HF ratio reflects the sympathovagal balance, which is usually interpreted as reflecting the relative sympathetic contribution to the control of heart rate. The increase of LF/HF ratio due to the training program might therefore reflect the increased LF power as adaptation to a more active lifestyle of the patients.

Impairment of frequency domain HRV measures in PAH patients has previously been demonstrated by Mc Gowan and colleagues (36). They concluded that a combination of diminished sympathetic neural heart rate modulation and increased right atrial stretch may attenuate HRV in PAH patients.

Our observed partial recovery of functional activity level dependent measurements of HRV due to exercise program 
Table 3 Activity level depending training effects on cardiac autonomic regulation (accelerometry) (values are mean \pm standard deviation)

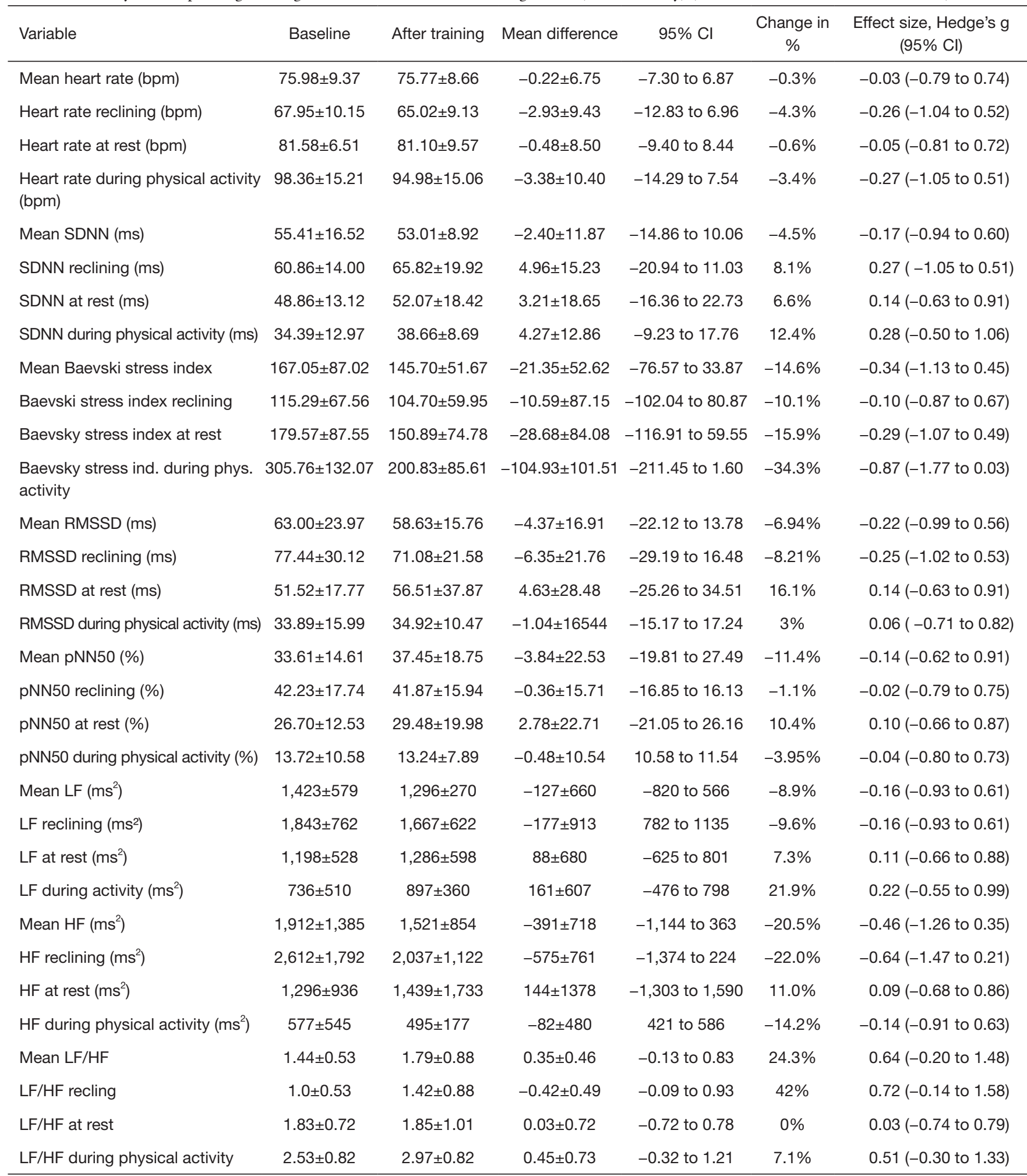

SDNN, standard deviation of normal-to-normal; LF, low frequency; HF, high frequency. 


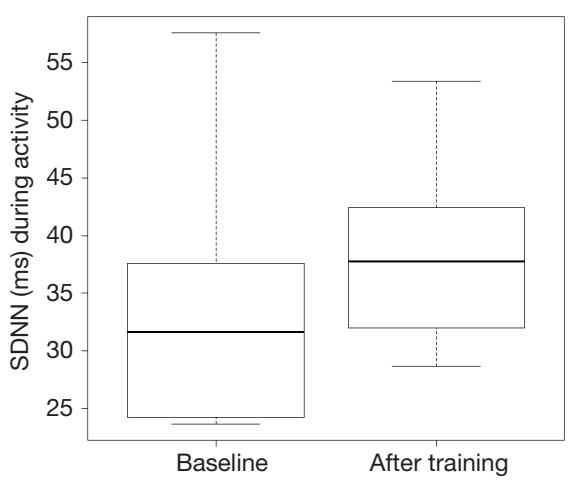

Figure 1 Box plot demonstrating the increase of Standard deviation of normal-to-normal intervals (SDNN) during activity due to the exercise program (Hedge's $g=0.28$ ).

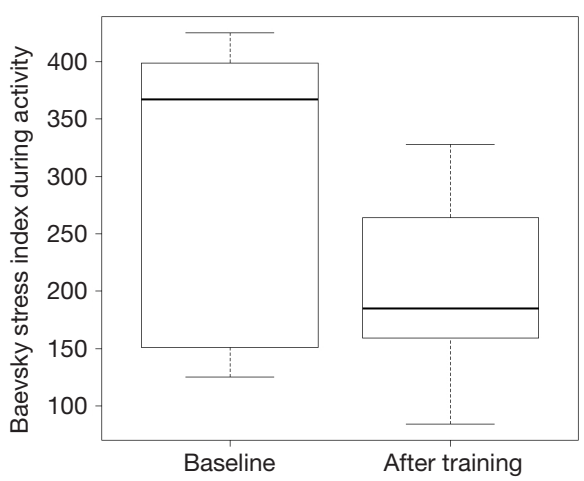

Figure 2 Box plot showing clinically meaningful changes of Baevsky stress index (BSI) during activity due to the exercise program (Hedge's $g=-0.87$ ).

may help to further explore the mechanisms of exercise training effects in PAH patients. Future studies might include analysis of longer sequences of cardio intervals that may enhance reproducibility of the assessment of regulatory mechanisms and its relation to the extent of physical activity. In particular, longer recording periods are helpful to obtain more reliable time-domain values (37), and should focus on the estimation of functional mechanisms of vegetative regulation under different activity levels such as activities of daily living. Parameters of cardiac autonomic regulation (i.e., stress indices) might be of specific diagnostic interest and potentially may serve for training control as for instance training memory, heart rate target zones and with large-scale functions in individual training counseling and training supervision.

\section{Study limitations}

The small number of patients studied and varying PAH symptoms may limit the interpretation of the data. However, to reduce heterogeneity we included only patients with IPAH or IPAH-similar disease, i.e., PAHCHD after shunt closure. In addition, although there was a longer time interval between the HRV measurement and the invasive assessment of hemodynamics, the patients' clinical condition remained stable and medical treatment unchanged, suggesting that the severity of PAH did not change significantly during that time.

\section{Conclusions}

According to the data of this pilot-study, a sixteen-week endurance and resistance training program in children and adolescents with $\mathrm{PAH}$ as add-on to optimized medical therapy resulted in clinical meaningful changes of activation of the sympathetic section of the vegetative nervous system. The analysis of these changes may help to understand the functional adaptation of the human organism under varying stress conditions associated with pulmonary vascular disease. Our results may serve for sample size calculation of further studies which are necessary to assess the relevance of these findings and its impact on the effects of exercise training in $\mathrm{PAH}$ patients.

\section{Acknowledgments}

We would like to thank all patients who participated in this study for their high motivation and the parents for their trust and support.

Funding: None.

\section{Footnote}

Provenance and Peer Review: This article was commissioned by the editorial office, Cardiovascular Diagnosis and Therapy for the series "Pediatric Pulmonary Hypertension". The article has undergone external peer review.

Data Sharing Statement: available at http://dx.doi. org/10.21037/cdt-20-263

Conflicts of Interest: All authors have completed the ICMJE uniform disclosure form (available at http://dx.doi. org/10.21037/cdt-20-263). The series "Pediatric Pulmonary 
Hypertension" was commissioned by the editorial office without any funding or sponsorship. CA served as the unpaid Guest Editor of the series. The authors have no other conflicts of interest to declare.

Ethical Statement: The authors are accountable for all aspects of the work in ensuring that questions related to the accuracy or integrity of any part of the work are appropriately investigated and resolved. The study was conducted in accordance with the Declaration of Helsinki (as revised in 2013). The study was approved by the Ethical Review board of the Justus-Liebig-University Giessen (approval number 219/13) and informed consent was taken from all the patients and caregivers.

Open Access Statement: This is an Open Access article distributed in accordance with the Creative Commons Attribution-NonCommercial-NoDerivs 4.0 International License (CC BY-NC-ND 4.0), which permits the noncommercial replication and distribution of the article with the strict proviso that no changes or edits are made and the original work is properly cited (including links to both the formal publication through the relevant DOI and the license). See: https://creativecommons.org/licenses/by-nc-nd/4.0/.

\section{References}

1. Berger RMF, Beghetti M, Humpl T, et al. Clinical features of paediatric pulmonary hypertension, A registry study. Lancet 2012;379:537-46.

2. Latus H, Delhaas T, Schranz D, et al. Treatment of pulmonary arterial hypertension in children. Nat Rev Cardiol 2015;12:244-54.

3. Barst RJ, McGoon MD, Elliott CG, et al. Survival in childhood pulmonary arterial hypertension: insights from the registry to evaluate early and long-term pulmonary arterial hypertension disease management. Circulation 2012;125:113-22.

4. Ivy DD, Rosenzweig EB, Lemarié J, et al. Long-term outcomes in children with pulmonary arterial hypertension treated with bosentan in real-world clinical settings. Am J Cardiol 2010;106:1332-8.

5. van Loon RL, Roofthooft MT, Delhaas T, et al. Outcome of pediatric patients with pulmonary arterial hypertension in the era of new medical therapies. Am J Cardiol 2010;106:117-24.

6. Haworth SG, Hislop AA. Treatment and survival in children with pulmonary arterial hypertension: the UK
Pulmonary Hypertension Service for Children 2001-2006. Heart 2009;95:312-7.

7. Ehlken N, Lichtblau M, Klose H, et al. Exercise training improves peak oxygen consumption and haemodynamics in patients with severe pulmonary arterial hypertension and inoperable chronic thrombo-embolic pulmonary hypertension: a prospective, randomized, controlled trial. Eur Heart J 2016;37:35-44.

8. Becker-Grünig T, Klose H, Ehlken N, et al. Efficacy of exercise training in pulmonary arterial hypertension associated with congenital heart disease. Int J Cardiol 2013;168:375-81.

9. Chan L, Chin LMK, Kennedy M, et al. Benefits of intensive treadmill exercise training on cardiorespiratory function and quality of life in patients with pulmonary hypertension. Chest 2013;143:333-43.

10. Fox BD, Kassirer M, Weiss I, et al. Ambulatory rehabilitation improves exercise capacity in patients with pulmonary hypertension. J Card Fail 2011;17:196-200.

11. Grünig E, Lichtblau M, Ehlken N, et al. Safety and efficacy of exercise training in various forms of pulmonary hypertension. Eur Respir J 2012;40:84-92.

12. Grünig E, Maier F, Ehlken N, et al. Exercise training in pulmonary arterial hypertension associated with connective tissue diseases. Arthritis Res Ther 2012;14:R148.

13. de Man FS, Handoko ML, Groepenhoff H, et al. Effects of exercise training in patients with idiopathic pulmonary arterial hypertension. Eur Respir J 2009;34:669-75.

14. Mereles D, Ehlken N, Kreuscher S, et al. Exercise and respiratory training improve exercise capacity and quality of life in patients with severe chronic pulmonary hypertension. Circulation 2006;114:1482-9.

15. von Scheidt F, Meier S, Krämer J, et al. Heart Rate Response During Treadmill Exercise Test in Children and Adolescents With Congenital Heart Disease. Front Pediatr 2019;7:65.

16. Wensel R, Jilek C, Dörr M, et al. Impaired cardiac autonomic control relates to disease severity in pulmonary hypertension. Eur Respir J 2009;34:895-901.

17. Latus H, Bandorski D, Rink F, et al. Heart Rate Variability is Related to Disease Severity in Children and Young Adults with Pulmonary Hypertension. Front Pediatr 2015;3:63.

18. Lammers AE, Munnery E, Hislop AA, et al. Heart rate variability predicts outcome in children with pulmonary arterial hypertension. Int J Cardiol 2010;142:159-65.

19. Witte C, Meyer-Arebnd JU, Andrié R, et al. Heart Rate Variability and Arrhythmic Burden in Pulmonary 
Hypertension. Adv Exp Med Biol 2016;934:9-22.

20. Paula-Ribeiro M, Ribeiro IC, Arnada LC, et al. Carotid chemoreflex activity restrains post-exercise cardiac autonomic control in healthy humans and in patients with pulmonary arterial hypertension. J Physiol 2019;597:1347-60.

21. van de Vegte YJ, Tegegne BS, Verweij N, et al. Genetics and heart rate response to exercise. Cell Mol Life Sci 2019;76:2391-409.

22. Aubert AE, Seps B, Beckers F. Heart rate variability in athletes. Sports Med 2003;33:889-919.

23. De Meersman RE. Heart rate variability and aerobic fitness. Am Heart J 1993;125:726-31.

24. Halliday SJ, Shi H, Brittain EL, et al. Reduced free-living activity level in pulmonary arterial hypertension patients. Pulm Circ 2019;9:2045894018814182.

25. Sehgal S, Chowdhury A, Rabih F, et al. Step- count using an Accelerometer for Monitoring PAH-STAMP Study group. Lung 2019;197:501-8.

26. Pugh ME, Buchowski MS, Robbins IM, et al. Physical activity limitation as measured by accelometry in pulmonary arterial hypertension. Chest 2012;142:1391-8.

27. Zöller D, Siaplaouras J, Apitz A, et al. Home Exercise Training in Children and Adolescents with Pulmonary Arterial Hypertension: A Pilot Study. Pediatr Cardiol 2017;38:191-8.

28. Dubowy KO, Baden W, Bernitzki S, et al. A practical and transferable new protocol for treadmill testing of children and adults. Cardiol Young 2008;18:615-23.

29. R Core Team (2015). R: A language and environment for statistical computing. R Foundation for Statistical

Cite this article as: Siaplaouras J, Frerix M, Apitz A, Zöller D, Apitz C. Effects of exercise training on heart rate variability in children and adolescents with pulmonary arterial hypertension: a pilot study. Cardiovasc Diagn Ther 2021;11(4):1028-1036. doi: $10.21037 /$ cdt-20-263
Computing, Vienna, Austria.

30. Danielak BA, Pruzek RM, Doane WE, et al. (2014). granovaGG: Elemental Graphics for Analysis of Variance using ggplot2. R package version, 1.2.

31. Horner SM, Murphy CF, Coen B, et al. Contribution to heart rate variability by mechanoelectric feedback. Stretch of the sinoatrial node reduces heart rate variability. Circulation 1996;94:1762-7.

32. Opie LH. Cellular basis for therapeutic choices in heart failure. Circulation 2004;110:2559-61.

33. Baevsky RM, Bennett BS, Bungo MW, et al. Adaptive responses of the cardiovascular system to prolonged spaceflight conditions: assessment with Holter monitoring. J Cardiovasc Diagn Proced 1997;14:53-7.

34. Kleiger RE, Miller JP, Bigger JT, et al. Decreased heart rate variability and its association with increased mortality after acute myocardial infarction. Am J Cardiol 1987;59:256-62.

35. Reyes del Paso GA, Langewitz W, Mulder LJM, et al. The utility of low frequency heart rate variability as an index of sympathetic cardiac tone: a review with emphasis on a reanalysis of previous studies. Psychophysiology 2013;50:477-87.

36. McGowan CL, Swiston JS, Notarius CF, et al. Discordance between microneurographic and heart-rate spectral indices of sympathetic activity in pulmonary arterial hypertension. Heart 2009;95:754-8.

37. Nunan D, Sandercock GRH, Brodie DA. A quantitative systematic review of normal values for short-term heart rate variability in healthy adults. Pacing Clin Electrophysiol 2010;33:1407-17. 